\title{
PROPOSIÇÃO E MODELAGEM DE UMA REDE DE SENSORES SEM FIO DE BAIXO CUSTO
}

\author{
PEDRo HENRIQUE FERREIRA MACHADO ${ }^{1}$, LUIZ EdIVAL DE SOUZA ${ }^{1}$ \\ 1. Instituto de Engenharia de Sistemas e Tecnologia da Informação, Universidade Federal de Itajubá \\ Caixa Postal 50, 37500-903 Itajubá, MG, Brasil \\ E-mails: machado.pferreira@gmail.com, edival@unifei.edu.br
}

\begin{abstract}
This paper presents the development of a system consisting of a network using wireless technology for communication of sensors and actuators, known as Wireless Sensor Network (WSN), in home automation scenario. In order to analyze and validate the system uses Petri nets that allow the creation of a model representing the dynamics of the traffic of messages.
\end{abstract}

Keywords_Event Discrete Systems, Petri Nets, Wireless Sensor Network, Home Automation

Resumo- Apresenta-se neste trabalho o desenvolvimento de um sistema formado por uma rede utilizando a tecnologia sem fio para a comunicação de atuadores e sensores, conhecida como Rede de Sensores Sem Fio, em uma rede doméstica. De forma a analisar e validar o sistema utiliza-se Redes de Petri que permitem a criação de um modelo representando a dinâmica das mensagens do sistema.

Palavras-chave— Sistemas a eventos discretos, Redes de Petri, Redes de Sensores Sem Fio, Domótica.

\section{Introdução}

Rede de sensores sem fio (RSSF) ou em inglês Wireless Sensor Networks (WSN) consiste em redes de dispositivos sensores que monitoram condições físicas ou comportamentos específicos, como exemplo temperatura, pressão, presença de objetos, etc.

Esta rede é caracterizada pelo uso de uma quantidade grande de nós-sensores com a capacidade de se comunicar (Rocha, 2007). O conjunto denominado nós-sensores, realiza além de sensoriamento, processamento de dados e comunicação com outros componentes da rede.

A comunicação entre os nós é feita através de uma topologia Ad-Hoc (Rodrigues, 2009). Nesta topologia, todos os dispositivos da rede funcionam como roteadores, encaminhando continuamente informações que vêm de dispositivos vizinhos.

Com estas características, os nós podem ser colocados próximos ao fenômeno analisado, permitindo assim uma arquitetura de controle de processos distribuída e flexível.

Esta tecnologia de comunicação tem ganhado muito espaço devido ao seu baixo custo de implementação e sua flexibilidade de aplicações.

Para tanto, segue neste trabalho uma proposta de uma arquitetura simples e funcional de um sistema RSSF de baixo custo.

Este artigo está organizado da forma descrita a seguir. Inicialmente, indica-se as tecnologias que norteiam o mercado das redes de sensores sem fio. Em seguida, tem-se a caracterização do sistema RSSF de baixo custo seguido da modelagem da comunicação do sistema. Por fim faz-se algumas análises do módulo proposto e da modelagem do sistema.

\section{Tecnologias de mercado}

\subsection{Cenário das Redes de Sensores Sem Fio}

Com a constante melhoria dos processos, expansão de plantas, necessidade de controle distribuído e baixo custo, a indústria vê com bons olhos $\mathrm{o}$ desenvolvimento da tecnologia wireless.

Neste ponto, trabalha-se a tecnologia das redes de sensores sem fio (RSSF). Esta é uma tecnologia emergente com amplas aplicações de conectividade com o meio físico. Isto torna possível a extração de informações vinculadas a um determinado ambiente de forma mais simples e mais flexível do que as técnicas de monitoramento e instrumentação tradicionais (Silva, 2006) que utilizam cabos e comunicação ponto-a-ponto. Seguindo a tendência da indústria, a automação residencial, ou domótica ou ainda home automation (Ryan, 1989), desenvolve cada vez mais em plataformas de comunicação RSSF de baixo custo.

Esta tecnologia de redes de sensores sem fio tem ganhado espaço uma vez que reduz consideravelmente os custos de hardware e praticamente elimina os gastos com cabeamento.

\subsection{Tecnologias RSSF industriais}

Comercialmente, duas tecnologias baseadas no conceito RSSF já estão inseridas no mercado industrial. São elas WirelessHart e ISA 100.11a.

A WirelessHART é baseada no protocolo de comunicação HART e na camada de aplicação do modelo OSI (Open Systems Interconnection). Esta tecnologia provê um protocolo robusto de comunicação wireless para um grande conjunto de aplicações de medições, controle e gerenciamento (HART, 2009) 
Sobre um critério um pouco mais flexível das aplicações de uma rede de sensores sem fio, a ISA 100.11a, desenvolvida pela International Society of Automation (ISA), não se prende a um protocolo específico (Costa et. al, 2012). Esta trabalha sobre diversos padrões de comunicação abertos com IPv6 e UDP. Além disso, o padrão ISA 100.11a utiliza a norma IEEE 802.15, que define a camada física e camada controle de acesso.

\subsection{Norma IEEE 802.15}

A norma IEEE 802.15 e suas subdivisões definem o padrão de rede de área pessoal sem fios (WPAN), onde se prevê a ligação entre dispositivos distanciados até 300 metros. O padrão 802.15 existe para garantir as especificações de redes pessoais com baixa potência e custo reduzido.

Esta norma tem como características a flexibilidade, o baixo custo e o baixo consumo de energia. Para tanto, tem-se que este é um padrão adequado para a tecnologia RSSF para domótica.

\subsection{Domótica}

A domótica (em inglês home automation, smart home ou mesmo domotics) surge como um ramo dos Edifícios Inteligentes, mas com aplicação nas habitações. A palavra Domótica é o resultado da junção da palavra latina Domus, que significa Casa, com a palavra Robótica. A domótica procura fornecer conforto e conveniência aos lares domésticos. A idéia base é automatizar tarefas e rotinas de uma casa e ainda possibilitar a capacidade de interagir com a casa remotamente (Patrício, 2009). Assim sendo, é possível controlar luminosidade e temperatura de ambientes, gerenciar o consumo de energia, garantir a segurança residencial entre outras diversas aplicações.

As redes domésticas automatizadas ainda são um campo da ciência extremamente novo, não existindo um conjunto de protocolos, equipamentos e dispositivos padronizados. Muitos ainda são emprestados do ambiente industrial e predial (Abreu et.al., 2011).

Seguindo a estrutura das redes industriais atuais, o setor de home automation utiliza como meio de comunicação as redes de sensores sem fio. Os sistemas wireless dão maior flexibilidade e menor custo de implementação tornando-o ainda mais atrativo.

As soluções para este setor são inúmeras, e os fabricantes, por vezes, evitam a interoperabilidade de sistemas a fim de garantir certo monopólio das aplicações. Contudo, no âmbito de uma padronização, um conjunto de empresas trabalha para uma maior conectividade entre seus equipamentos. Neste sentido, tem-se a ZigBee Alliance, a qual com seu conjunto de associados busca a interoperabilidade dos produtos (ZigBee Alliance,
2011). Dá-se um destaque para esta associação uma vez que esta agrega duas tendências de mercado: utilização de RSSF e a interoperabilidade dos dispositivos.

\section{Descrição da proposta}

Em linhas gerais, este trabalho tem por funções modelar e desenvolver uma pequena rede de sensores sem fio de baixo custo visando sua aplicação em sistemas de automação residencial.

Para tanto, faz-se aqui uma proposição de um módulo composto por um microcontrolador, um rádio de comunicação e um elemento sensor de custos reduzidos. Em seguida, tem-se a modelagem de uma arquitetura de comunicação, representada graficamente por redes de Petri.

\subsection{Caracterizações do módulo}

As redes de sensores sem fio estão intimamente conectadas com a topologia de controle descentralizado. A chave que conecta estas duas das atuais vertentes da automação é, justamente, a autosuficiência dos dispositivos presentes na rede.

Esta auto-suficiência, que permite aos dispositivos funcionarem com certa independência dos outros dispositivos da rede, é dada pela junção da capacidade de processamento do microcontrolador, permitindo aquisição e tratamento de variáveis de sensores e atuadores, com os rádios transceptores que permitem a comunicação com outros dispositivos sem fio.

Para o sistema RSSF proposto neste trabalho, cria-se dois tipos distintos de dispositivos autosuficientes: o nó-sensor e o nó-registrador.

O nó-sensor tem duas funções bem definidas: controle do subsistema e comunicação. A primeira função é ler algumas variáveis de um dado processo (subsistema), avaliá-los e retornar uma ação de controle. A segunda é estabelecer comunicação com outros dispositivos da rede.

Já o nó-registrador tem quatro funções: comunicação, armazenamento, processamento e ajuste de parâmetros. A comunicação e processamento assemelham-se as funções do nósensor. A função de armazenamento é registrar os dados vindos dos nós-sensores (subsistemas). Por fim, o ajuste de parâmetros é repassar informações de alto nível de controle aos subsistemas como diagnóstico de rede e parametrização das funcionalidades dos nós-sensores. 


\section{Estudo de Caso}

Tem-se aqui um estudo de caso de um sistema de sensoriamento aplicado a domótica utilizando RSSF. O sistema como já descrito, possui dois tipos de nós: o sensor e o registrador. O primeiro é um subsistema de controle de temperatura ou de luminosidade, podendo haver inúmeros nós-sensores. $\mathrm{O}$ registrador é único nesta rede, e desempenha as funções já descritas.

O módulo físico do nó-sensor é um conjunto de um micrcontrolador, um rádio transceptor e um elemento sensor. Para o nó-registrador, tem-se um rádio transceptor, uma interface rádio-computador e um computador.

\subsection{Módulos do Sistema}

Seguindo a proposta deste trabalho, utiliza-se dispositivos de baixo custo na construção dos módulos de nó-sensor e nó-registrador.

Para o nó-sensor utiliza-se um microcontolador ATMEGA328-PU da empresa Atmel. O seu baixo custo, a facilidade de programação e grande versatilidade de programação foram os critérios utilizados para sua aplicação neste trabalho. $\mathrm{O}$ rádio de comunicação é o SHUNCOM SZ05-ADV que se comunica serialmente com outros rádios e com a interface do computador. Este também segue os critérios de baixo custo e facilidade de implementação. Como elementos sensores, têm-se sensores de temperatura LM35 do fabricante National Semiconductor e sensor de luminosidade LDR de fabricação da SUNROM. Como atuadores tem-se potenciômetros digitais da Microchip capazes de ajustar a intensidade de luz de um conjunto de leds e o ar-condicionado dos ambientes.

O hardware do sistema do nó-sensor é estruturado da seguinte forma, como se segue na figura 1:

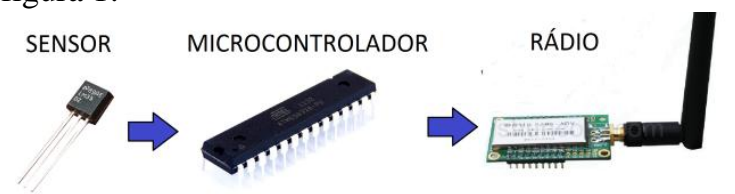

Figura 1: Módulo dos nós-sensores

- Sensor de luminosidade: sistema fotossensível que utiliza a variação da resistência segundo a intensidade de luminosidade;

- Sensor de temperatura: sistema termosensível que tem a resistência variando segundo a temperatura;

- Microcontrolador: processa o dado recebido do sensor, calcula e controla a variável de interesse do processo. Além disso tem por funde encapsular os frames das mensagens;

- Rádio: transmite os frames (informações) advindos do respectivo nó-sensor.
O módulo do nó-registrador tem um hardware mais simples, utilizando apenas um rádio transceptor (SZ05-ADV), uma interface RS-485 para comunicação com o computador e um computador com um software supervisório. Esta disposição do nó-registrador permite que este receba os dados de um dado subsistema (nó-sensor) e os armazene. Além disso, esta estrutura permite enviar aos nóssensores mensagens de ajuste, parametrizações, avaliação da rede e diagnósticos de rede.

Sendo assim, após a definição do hardware, evidencia-se uma estrutura da rede na figura 2.

Nó-Sensor

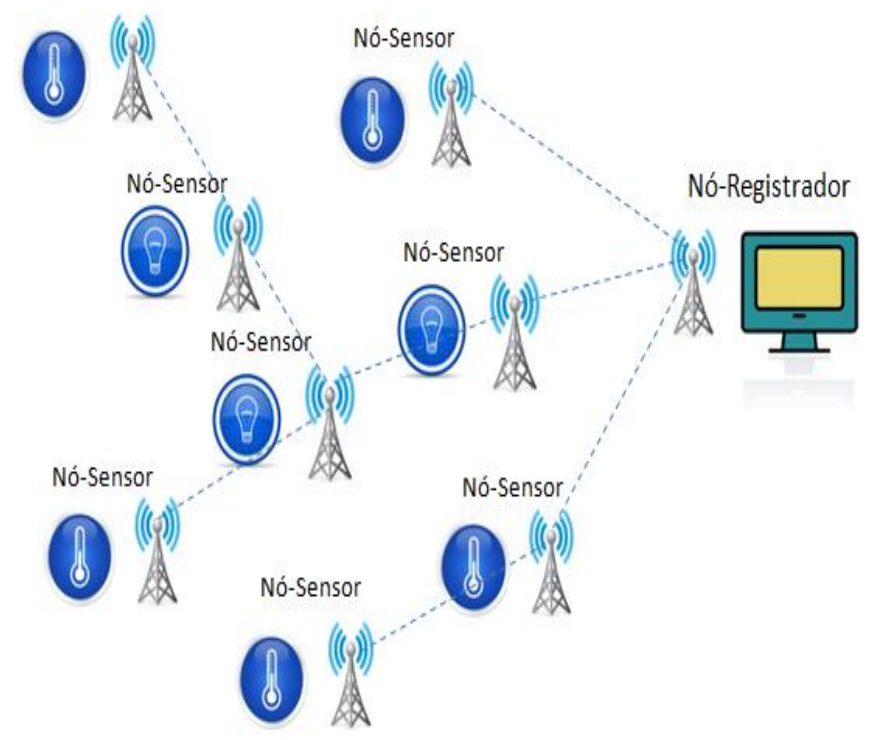

Figura 2: Sistema proposto para o nó-sensor

\subsection{Protocolo de Comunicação}

Após estruturado a arquitetura do sistema, desenvolve-se estrutura-se um protocolo de comunicação que será estabelecido entre os nós da rede.

O protocolo estabelece as seguintes funções: a descoberta de nós, gestão de ligações, tratamento de rotas, envio e recepção de frames. A estruturação de funcionamento do protocolo da rede é baseada em (Rodrigues, 2009).

Ele funciona uma camada acima da aplicação, ou seja, este permite que os subsistemas de nóssensores se comuniquem e criem, de fato, a arquitetura da rede de sensores sem fio.

Neste protocolo, inicialmente estabelece-se as rotas entre os nós. Para isto, envia-se uma mensagem do tipo RANN (Root Annoucement), o qual tem por função indicar a presença deste nó na rede. Em seguida uma mensagem do tipo PREQ (Path Request) envia uma requisição para estabelecer conexão com o nó raiz. Por conseguinte, tem-se que o nó raiz envia PREP (Path Reply) com estabelecendo a comunicação entre os nós. Na figura 3 , tem-se um fluxograma para exemplificar a estrutura. 


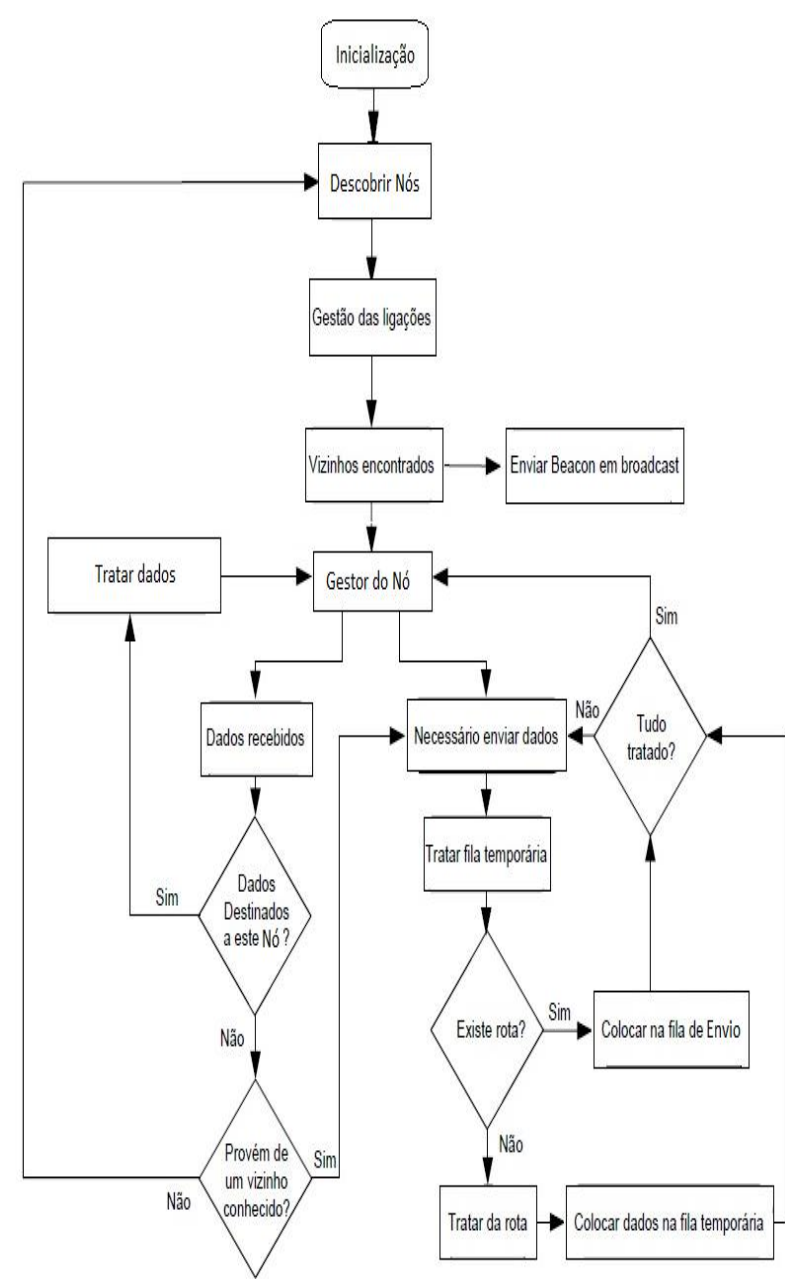

Figura 3: Fluxograma do Nó-Sensor

Os nós quando se ligam enviam uma mensagem de reconhecimento de rede (beacon frame). Seguidamente testa-se as ligações dos novos vizinhos, ativando a gestão das ligações que irá ficar ligada durante todo o funcionamento da rede. Quando a ligação entre vizinhos está ativa, é ativado um evento que envia sistematicamente beacons para assegurar que os vizinhos se encontram ativos. Como próximo passo, o nó entra no modo de envio/recepção de dados. Quando o nó quer enviar dados e já possui a rota ou é para enviar em broadcast coloca essa informação na respectiva fila, dependendo se é um frame de dados ou um frame de gestão. Quando não existe uma rota na tabela de roteamento, é feito um pedido para a mesma ser atualizada. O método que trata da tabela de roteamento encarrega-se de tentar encontrar a rota. Enquanto a rota é encontrada, esse frame é colocado numa fila temporária. Os dados que estão nesta fila são tratados sempre que é necessário enviar dados, ou seja, são verificados todos os frames que se encontram na fila temporária para ver se já existe uma rota para elas e então são enviadas.

Quando o nó recebe dados, este verifica à quem os dados são destinados. No caso de ter recebido uma mensagem destinado a ele, os dados são tratados e uma ação de controle ou diagnóstico é executada. No entanto, se o frame de dados não forem destinadas a ele, o nó verifica se tem rota para o destino e enviaas, ou coloca-as na fila temporária para posterior envio.

\subsection{Modelagem do protocolo através de rede de Petri}

Seguindo a proposta do trabalho, é evidenciado uma modelagem do protocolo de comunicação usando o formalismo de redes de Petri.

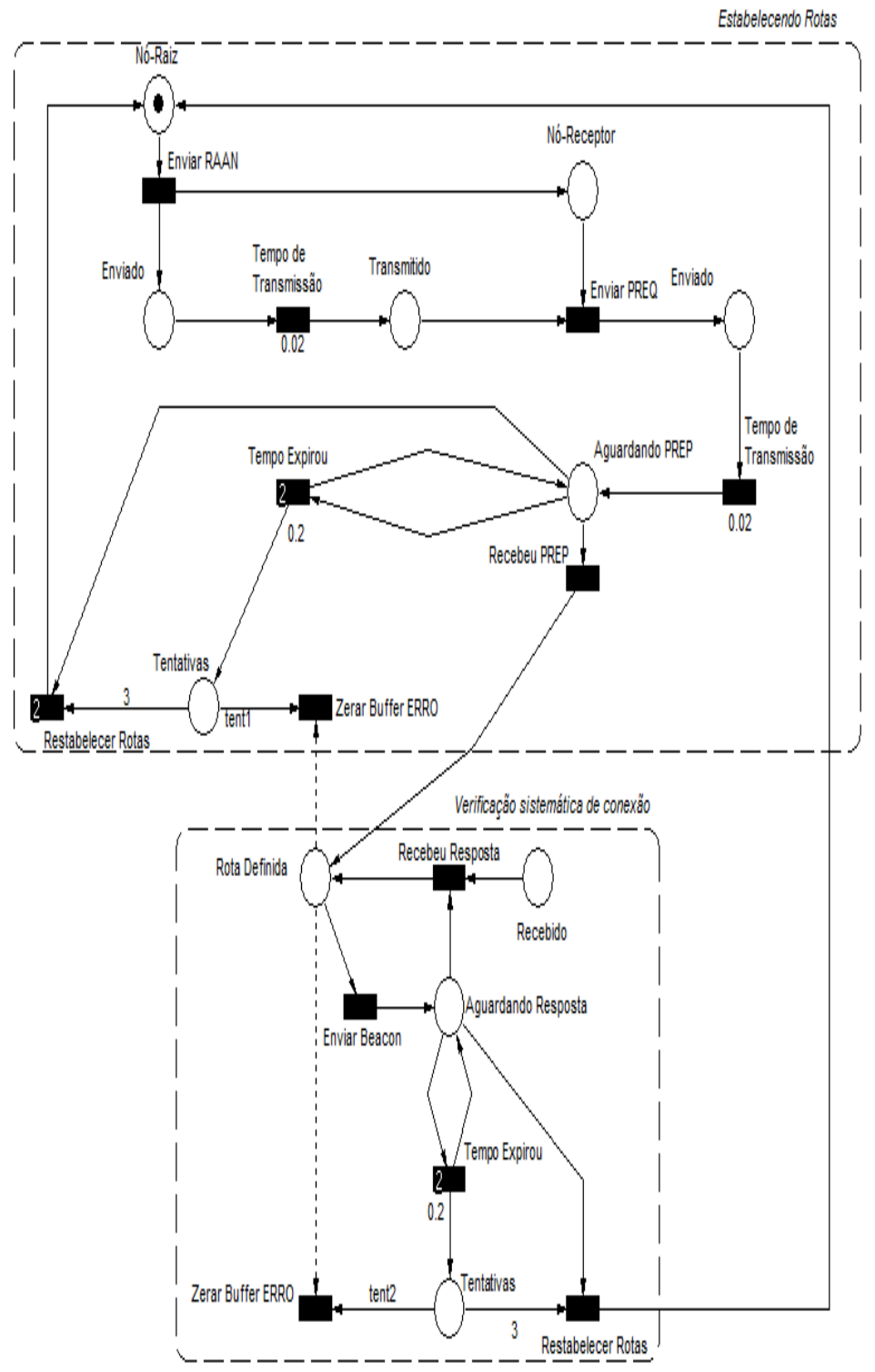

Figura 4: Modelo RdP para o modo de estabelecimento de rotas 


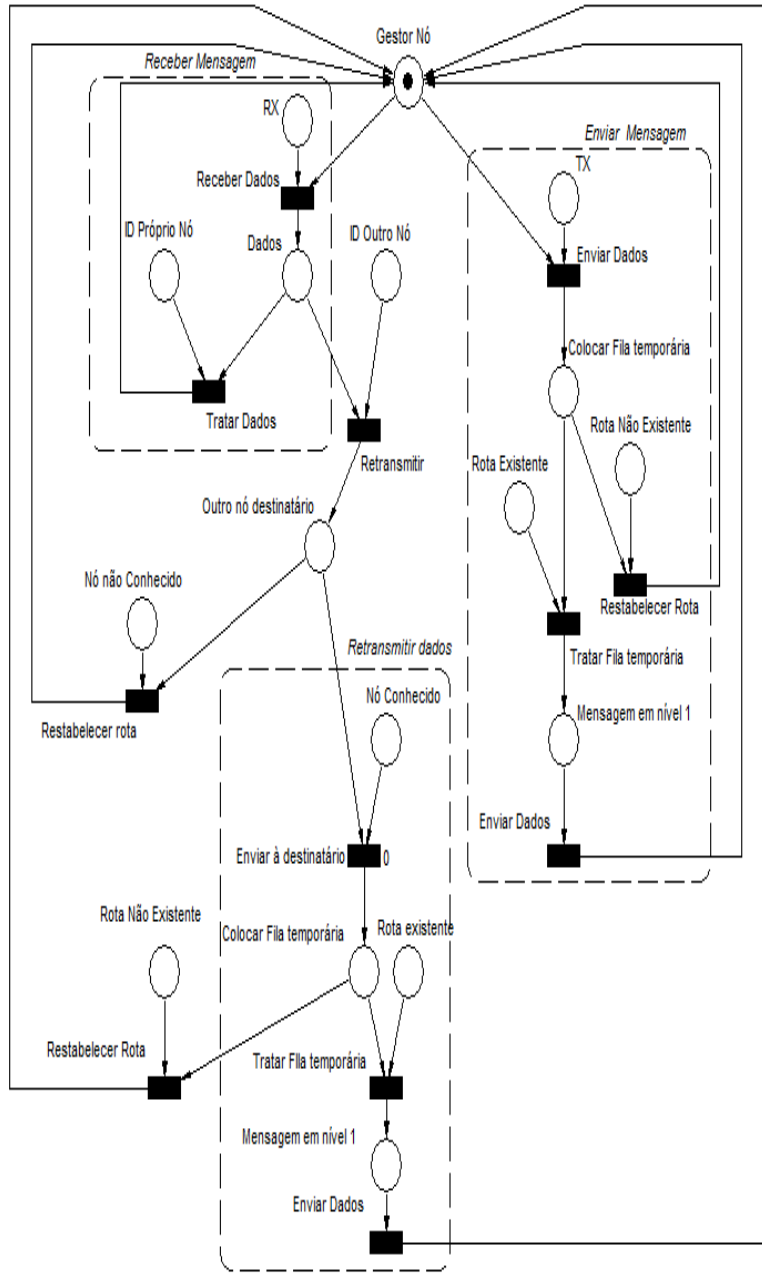

Figura 5: Modelo Rdp para o modo de envio/recebimento

A figura 4 indica a marcação inicial do modelo de busca de rotas, estabelecido em todos os dispositivos da rede. Há de se ressaltar que a marcação do lugar Recebido do submodelo Verificação sistemática de conexão é um artifício de simulação que recria um estado no qual o nó recebe a resposta da mensagem beacon.

O segundo modelo apresentado na figura 5 , evidencia a marcação inicial do comportamento do nó no recebimento e envio de dados. Os lugares $T X$, $R X, I D$ Outro Nó, ID Próprio Nó, Nó Conhecido, Nó Não Conhecido, Rota Existente e Rota Não Existente, são também artifícios de simulação para indicar escolhas e possibilidades do sistema, uma vez que neste nível de abstração do sistema não se detalha o programa do controlador no tratamento de dados.

\section{Análises}

Inicialmente há dê se salientar que este trabalho trata de uma proposta de um sistema de baixo custo, e claramente sua construção e fabricação precisa de aperfeiçoamentos.

Tendo isto em mente, percebe-se que o que fora desenvolvido neste trabalho contribui para a análise da dinâmica da rede de sensores sem fio utilizando módulos de baixo custo. Os modelos representados em rede de Petri sintetizam a arquitetura do protocolo de comunicação que deve ser estabelecido entre os nós.

Este tipo de formalismo permite apenas definir conceitos qualitativos da estrutura do sistema. A partir deste, pode-se evoluir a pesquisa no sentido de análises mais criteriosas de uma rede de dispositivos sensores, como por exemplo, colisões de mensagens, algoritmos de erro, tempo de transmissão, número de pacotes suportados e outras estocásticas que interferem nos sistemas RSFF.

\section{Conclusões}

Pela proposta do trabalho, verifica-se que estruturalmente o sistema de rede de sensores sem fio de baixo custo é aplicável no conceito da domótica. Com este trabalho, fica caracterizado uma metodologia para o desenvolvimento e aplicação de RSSF que pode ser implementado em dezenas de outras atividades de monitoração doméstica.

A modelagem em rede de Petri do sistema de comunicação, evidenciando um protocolo genérico de RSSF, apresentou-se interessante uma vez que é possível ter uma avaliação da dinâmica do conjunto de forma rápida e bastante conceitual.

Contudo, fica evidente também as limitações deste formalismo. Para uma análise mais criteriosa e mais quantitativa, por exemplo análise de tráfego, prioridade de acesso ao meio, check de erro este tipo de modelagem tem de se aplicar um nível mais alto de abstração. Para trabalhos futuros, o uso de outras ferramentas de modelagem como exemplo redes de Petri coloridas, são sugeridas.

Porém, de forma abrangente, tem-se que é possível a aplicação de redes de sensores sem fio de baixo custo no âmbito da home automation, e podese dizer ainda, que há grandes perspectivas de mercado para este setor.

\section{Agradecimentos}

O autor agradece a colaboração do órgão de fomento CAPES através das bolsas de pesquisa e o mestre Reinaldo Lima de Abreu pelo apoio técnico desta aplicação.

\section{Referências Bibliográficas}

Rocha, J. W. V. (2007) Rede de Sensores Sem Fio. Teleco - Inteligência em Telecomunicação. [Online] http://www.teleco.com.br/tutoriais/tutorialrssf/pagina _1.asp [Acessado: 25 de Maio de 2013].

Silva, I. M. D. (2006.) Redes de Sensores sem Fio aplicadas em Ambientes Industriais de Petróleo e Gás. Universidade Federal do Rio Grande do Norte [Online]: http://www.ccet.ufrn.br/prh22/trabalhos/graduacao/2 006/Ivanovitch_Silva_PRH22_UFRN_G.pdf

[Acessado em: 27 de Maio de 2013].

Ryan, J.L. (1989). Home Automation. Eletronics \& Communication Engeneering Journal, Vol.(4), pp. 185-192. 
Rodrigues, N. J. P. F. (2009). Redes Mesh Sem-Fios. Faculdade de Engenharia da Universidade do Porto. [Online]:http://paginas.fe.up.pt/ ee03172/files/tese_v ersao_provisoria_NunoRodrigues.pdf [Acessado: 28 de Maio de 2013].

Hart Communication Foundation (2009). Wireless

Hart Protocol. [Online] Disponível em:

http://www.hartcomm.org/protocol/training/training _ resources_wihart.html [Acessado: 28 de Maio de 2013].

Costa, M. S. e Amaral, J. L. M. (2012) Analysis of wireless industrial automation standards: ISA100.11a and WirelessHART. [Online] Disponível em:

http://www.isa.org/InTechTemplate.cfm?template=/ ContentManagement/ContentDisplay.cfm\&ContentI $\mathrm{D}=92650$. [Acessado em 30 de Maio de 2013]

IEEE Standarts Association (2005). IEEE 802.15 TM: WIRELESS PERSONAL AREA NETWORKS.

[Online] Disponível em:

http://standards.ieee.org/about/get/802/802.15.html

[Acessado em: 30 de Maio de 2013].

Murata, T. (1989). Petri Nets: Properties, Analysis and Applications,Proceedings of IEEE, Vol. 77-4.

Patrício, G. J. H. (2009). Rede Sem Fios de Microcontroladores com Acesso Remoto Aplicada à Domótica. Universidade Nova de Lisboa. [Online]: http://run.unl.pt/bitstream/10362/1977/1/Patricio_20 09.pdf [Acessado em 29 de Maio de 2013].

Abreu, E. R., Valim, P. R. O. (2011). Domótica: Controle de Automação Residencial Utilizando Celulares com Bluetooth. VIII SEGeT-Simpósio de Excelência em Gestão e Tecnologia.

ZigBee Alliance (2011). ZigBee Home Automation Overview. [Online] Disponível em: http://www.zigbee.org/Standards/ZigBeeHomeAuto mation/Overview.aspx [Acessado em 18 de Junho de 2013]. 